\title{
Sentiment Classification of Tweets in Twitter using CNN and Dropouts in RNN
}

\author{
Poornima A. \\ Post Graduate Student \\ PSG College of Technology \\ Coimbatore
}

\author{
K. Sathya Priya, PhD \\ Assistant Professor (SI.Gr) \\ PSG College of Technology \\ Coimbatore
}

\begin{abstract}
Sentimental analysis is the computational study of people's opinion, attitudes and emotions toward entities, individuals, issues, events or topic. A lot of research has been done to improve the accuracy of sentiment analysis, varying from simple linear models to more complex deep neural network models. Recently, Deep learning has shown great success in the field of sentiment analysis and is considered as the stateof-the- art model. The twitter data imposes many challenges, due to its complex structure, various dialects, in addition to the lack of its resources. Although, the recent Deep learning models have improved the accuracy of the twitter sentiment analysis, there is still more chance for improvement. This encouraged to explore different deep learning hybrid models that have not been applied to twitter data, in order to improve the twitter sentiment analysis accuracy. The objective of this paper is to improve the accuracy of sentiment analysis of twitter data by implementing a hybrid model of CNN-RNN techniques and by introducing dropout in the hybrid model and also compare the performance of the proposed method with existingmodels.
\end{abstract}

\section{General Terms}

Dropout, CNN, RNN

\section{Keywords}

Sentiment analysis, Text classification, Hybrid models, CNN, RNN, Dropouts, Accuracy

\section{INTRODUCTION}

Sentimental Analysis (SA) is a contextual mining of text which recognizes and extorts subjective information from the source material. Sentimental Analysis assists a business to comprehend the social sentiment of their service, brand or product even as observing online chats. SA manages sentiments, subjective text, and opinions. It is a method of knowing user's opinions, emotions and attitude subjected towards an item which can imply to events, topics or individualsof recent trends. Sentiment analysis can be performed in three levels namely sentence level, aspect level and document level. Twitter is a rich information source for decision making using sentiment analysis. Sentiment Analysis of brief informal texts on social media summarizes opinions as positive, neutral or negative statement of the opinion holder. Sentiment analysis over Twitter provides the firm an effective and rapid way to supervise the feelings of public towards a brand. As the platform of twitter uses tweets to denote opinions in sentence form, the sentence level SA is used for examining sentiments. The goal is to calculate the sentiment accuracy of sentences that were extracted from the text of tweets. The sentiment analysis of the tweet helps to find whether the sentiment of the tweet on particular products, events, etc., is positive or negative. This analysis help concerned organizations to find opinions of people about their product, events, so on from the tweets. The challenging aspect in sentiment analysis is an opinion word which is considered as a positive in one situation and may be considered as negative in another situation. The traditional text processing considers that a little change in two bits of content has no change in meaning. But in sentiment analysis a change in two words of content has change in meaning, for example, "The smell of the flower is good" is different from "the smell of the flower is not good". The sys- tem processes it by analyzing one sentence at a time. However, blogs and twitter contain more informal sentences which user can understand but system cannot understand it. This paper improves the performance of text classification by combining Convolutional Neural Network (CNN) and Recurrent Neural Network (RNN) and introducing dropout in the hybrid model. This paper is organized in such a way that the literature survey is discussed in section II, followed by the description of proposed work in section III, the result of sentiment analysis is analyzed in section IV and the conclusion in section $\mathrm{V}$.

\section{LITERATURE SURVEY}

In the opinion mining, sentiment analysis is the process of contextually mining text to identify and categorize the subjective opinions expressed by the writers. Normally it is used to determine whether the writer's attitude towards a particular topic or product, etc. is positive, negative, or neutral. Businesses use it to understand the social sentiment of their brand, product or services while monitoring online conversations. In the context of twitter, sentiment analysis quantifies the mood of a tweet or comment by counting the number of positive and negative words. By subtracting the negative comments from the positive comments, the sentiment score is generated.

Wazery et al [1] classified the tweets in twitter dataset into the people's opinion of different sentiment polarity. Here, the two main approaches for sentiment analysis were applied. The first approach uses machine learning algorithms like support vector machine, nave bayes, decision tree, and $\mathrm{K}$ - nearest neighbor and the second approach uses deep neural networka Recurrent Neural Network using Long Short-Term Memory (LSTM). Recurrent Neural Networks of LSTM was applied to classify the tweets into positive or negative polarity. The accuracy of this architecture was compared with some machine learning based approaches like SVM, KNN, Naive bayes and Decision tree. The authors proved that the RNNLSTM had achieved the highest accuracy at $88 \%$ and the Naive Bayes showed the lowest accuracy of $69 \%$. Also, Decision Tree and Support Vector Machine showed accuracy scores of $81 \%$ and $82 \%$ respectively for Amazon dataset. Similar tests were conducted for IMDB dataset and Airline 
dataset. The authors concluded that RNN-LSTM performs better in terms of accuracy when compared to other machine learning models for all the three datasets.

Iimania et al [2] applied Deep Neural Networks to solve aspect based sentiment analysis. Aspect matrix of the input sentence was calculated using the dense layer for bag of words input layer. Indonesia dataset was used for their experiment. They compared two deep neural network models - Gated Re- current Units (GRU) and fully connected layer with different inputs on aspect detection and sentiment classification. The result of aspect matrix is better than other word embedding approaches. This paper proved that GRU used for aspect detection of sentiment analysis performed better than fully connected layer.

Patel and Tiwari [3] performed sentiment analysis in IMDB movie review dataset using recurrent neural network. The performance of machine learning approaches was compared with recurrent neural network for sentiment classification. It was concluded that RNN has the best accuracy of $82 \%$ than other deep neural networks on movie review dataset of sequential information.

Thomas and Latha [4] performed sentiment analysis on tweets in south Indian language like Malayalam. Apart from English, SA has spread its wing to other natural languages which is significant in a multi linguistic country like India. Recurrent Neural Network - Long short term memory model (RNNLSTM) was used to predict the sentiment in tweets and achieved an accuracy of $80 \%$. It was suggested that the accuracy of the results can be improved by having different datasets.

Hassan and Mahmood [5] employed Recurrent Neural Network Long Short-Term Memory (RNN-LSTM), on top of pretrained word vectors for sentence-level classification tasks. They introduced a language model that utilizes only word2vec inputs over LSTM. They captured more information about the syntactics and semantics of words by using pre-trained word vectors and proposed a model to overcome several traditional methods like bag of words and n-gram model. The results proved that the proposed model achieved better performance than the traditional methods.

Zhang et. al [6] assigned correct polarity to a given sentence by considering the entity on which an opinion is expressed by using RNN-LSTM and Gated Recurrent Unit (GRU). A novel co-attention based network was proposed to capture the relationship between aspect term and its surrounding contexts. This network addresses the "target-sensitive sentiment" issue in the Apsect based sentiment analysis (ABSA) task. This model achieved parallelized training and can be applied to address large scale datasets with limited training costs.

Zhang et. al [7] developed a new architecture based on termed Comprehensive Attention Recurrent Neural Networks (CARNN) which can store preceding, succeeding and local contexts of any position in a sequence. Bidirectional recurrent neural networks (BRNN) was used to access the past and future information. The RNN is also replaced by long shortterm memory (LSTM) and gated recurrent unit (GRU), to enhance the effectiveness of the new architecture. The results on the IMDB Large Movie Review Dataset demonstrate that the new architecture was superior to recurrent models and the bidirectional recurrent models in terms of classification accuracy. One problem of recurrent models is that the training procedure is time-consuming.

Yang et. al [8] demonstrated that the performance of tradi- tional text sentiment analysis method is based on the quality of feature extraction. The text sentiment analysis method based on RNN can obtain context information and CNN can obtain important features of text through pooling, but it is difficult to obtain context information. RNN and CNN was combined in different way and the sub-analysis network of CRNN and R-CNN was constructed. Then, these two networks were combined through the fusion gating unit. Thus the better accuracy was achieved and can capture the emotional features of review text better.

Du and Huang [9] proposed a bi-directional recurrent neural network algorithm based on the neural network attention mechanism for text classification. The feature vector was fed to a softmax classifier. They used two datasets for the text classification of bi-directional recurrent neural network. The classification accuracy of the proposed model was approximately $88.5 \%$ and it outperformed the traditional system.

Abdiaet. al [10] presented a deep-learning based method to classify user's opinion expressed in reviews (called RNSA). RNN-Long Short-Term Memory (LSTM) was employed for sequential processing and to overcome several shortcomings of traditional methods. Furthermore, sentiment knowledge, sentiment shifter rules and multiple strategies were used to overcome word coverage limit of an individual lexicon and word sense variations. To improve the effectiveness of the method sentence level, sentiment classification on large scale movie review datasets was conducted.

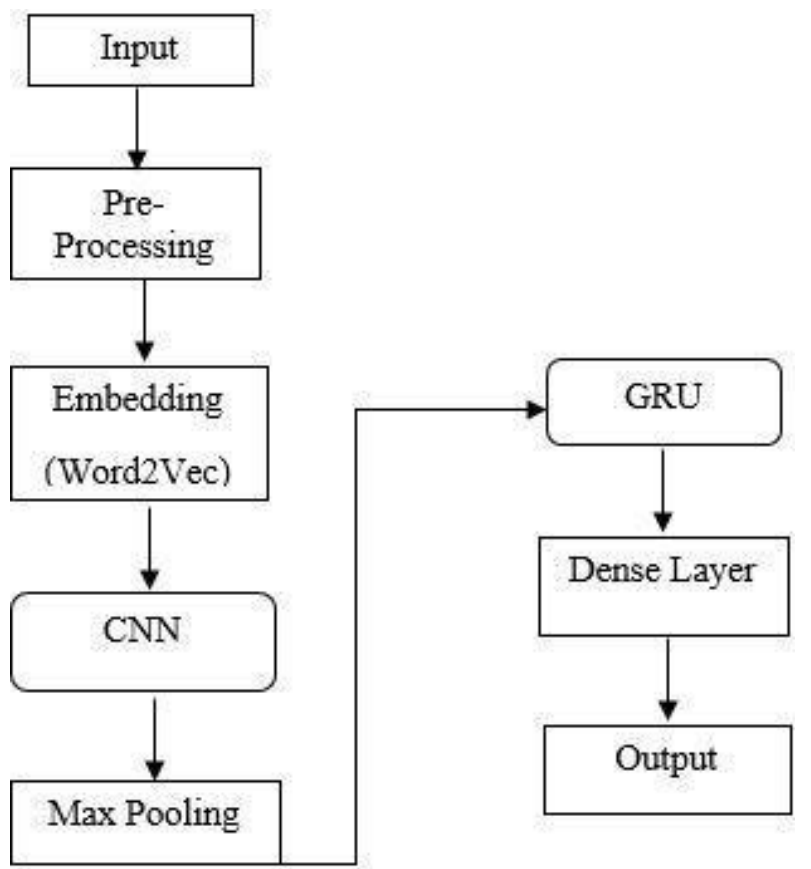

Fig 1: Proposed methodology

\section{PROPOSED WORK}

Sentiment analysis is performed to classify the sentence in tweets by combining the CNN-RNN models. Then the performance of the hybrid model is analyzed.

\subsection{Dataset Description}

The data is in comma-separated files with tweets and their corresponding sentiments. Tweet represent the text opinion about the product or event. The training dataset is a csv file which contains tweetid, sentiment, time and date when the tweets has been posted. The dataset also contains group of words, emoticons, symbols, URLs and references to people. 
The words are also a mixture of misspelled words, extra punctuations, and words with many repeated letters. So the data has to be preprocessed to convert it into a standard form suitable for classification.

\subsection{Preprocessing}

Raw tweets scraped from twitter generally result in a noisy dataset. This is due to the casual nature of people's usage of social media. Raw twitter data has to be normalized to create a dataset that can be learned by various classifiers. Preprocessing of data was done to standardize the dataset and reduce its size. The training and testing of the classifier was done using preprocessed dataset. Tweets have certain special characteristics such as retweets, emoticons, user mentions, etc. which have to be suitably extracted. The noisy data or special characters are removed by lemmatization, stop word removal, etc., Other preprocessing that are carried out in this work are converting the tweet to lower case, replacing 2 ormoredots (.) with space, strip spaces and quotes ("and ') from the ends of tweet, replacing 2 or more spaces with single space, etc., For example, consider the sentence, "i like the product!. After preprocessing the resultant sentence is " $\mathrm{i}$ like product".

\subsection{Word Embedding Model}

Word2Vec is one of the most popular techniques to learn word embeddings using shallow neural network. Gensimimplementation of Word2Vec is used. The first step is to prepare the text corpus for learning the embeddings by creating word tokens, removing punctuation, removing stop words, etc., Word2vec() method is used in order to train the word2vec model on the corpus. By this method, the embedding vectors of the preprocessed data can be produced. The few important arguments are used for embeddingmethod:

1) Sentences: List of sentences; here the list of reviewsentences is passed.

2) Size: The number of dimensions in which a word is represented. This is the size ofthe word vector.

3) Min count: Word with frequency greater than min count are only going to be included into the model. Usually, the bigger and more extensive thetext, the higher this number canbe.

4) Window: Only terms that occur within a windowneighborhood of a term, in a sentence, are associated with it during training. The usual value is 4 or5.

5) Workers: Number of threads used in training parallelization, to speed uptraining.

6) Dim: Dimension of word vector. Here 100is used.

7) Maxlen: Maximum number of the featurevectors.

\subsection{Feature Extraction}

Convolution Neural Network $(\mathrm{CNN})$ is a special kind of multi-layer neural network which consists of one or more convolutional, pooling layers, and dropout followed by one or more fully-connected layers. The convolutional and pooling layers implicitly extract relevant feature representation from input data, and fed it tothe fully connected layers for classification. The size and weights of the convolution filters determine the features to be extracted from the input data. Same convolution filter is floated overthe complete input data in order to extract similar features at different spatial locations. Max pool layer is then applied to select the most significant features from the CNN features. Subsequently, after iterating several convolutional and max pooling layers, it is fed to RNN model for classification. In general, then use softmax as an activation function in thefully connected layer. The parameters of $\mathrm{CNN}$ are filters, strides, kernel size, padding and activation. The few important arguments of CNN are Filters $=25$, Kernel size $=3$,Padding $=$ 'valid', Strides $=1$, Activation $=$ 'relu' are used in the experiment.

\subsection{Sentiment Analysis of Sentiment Classification}

A recurrent neural network (RNN) is a class of artificial neural network where connections between nodes form a directed graph along a sequence that helps in various tasks. This allows it to exhibit dynamic temporal behavior for a time sequence. RNNs are neural networks that add additional weights to the network to create cycles in the network, in an effort to model time dependencies and sequential events. The pre-trained embedding Word2Vec is used. RNN is complex to understand, it is quite interesting. It encapsulates a very beautiful design that overcomes traditional neural network's shortcomings that arise when dealing with sequence data: text, time series, videos, DNA sequences, etc. RNN is a sequence of neural network blocks that are linked to each other's like a chain that can be used to process text data, which is a sequence type. The order of words is very important to the meaning. Hopefully RNN can take care of word sequence and also capture term dependencies. Gated Recurrent Unit (GRU) is a type of RNN model and it is used to classify the text data from the CNN layers. The GRU layer consists of hidden dimension $=100$, fully connected layer $=1$, dropout rate $=0.2$, learning rate $=0.001$, batch size $=$ 50respectively.

\section{RESULTS AND DISCUSSION}

The result is analyzed by combining CNN and RNN models for sentiment analysis of text classification. The CNN and RNN were tested individually on Twitter dataset obtained from kaggle. The dataset consists of four attributes and 1.6 million instances. Accuracy has been calculated for both models and to combine the two models to get better accuracy than these two. The comparison of accuracy of classification of twitter between two different deep learning models and hybrid model are calculated and shown in figure 3. The accuracy forCNNmodel is approximately $78.64 \%$, $\mathrm{RNN}$ is $75.33 \%$ and the hybrid model of CNN-RNN is $81.90 \%$. Then, the hybridmodel of CNN-RNN outperforms the individual classification models with an accuracy of $81.90 \%$.

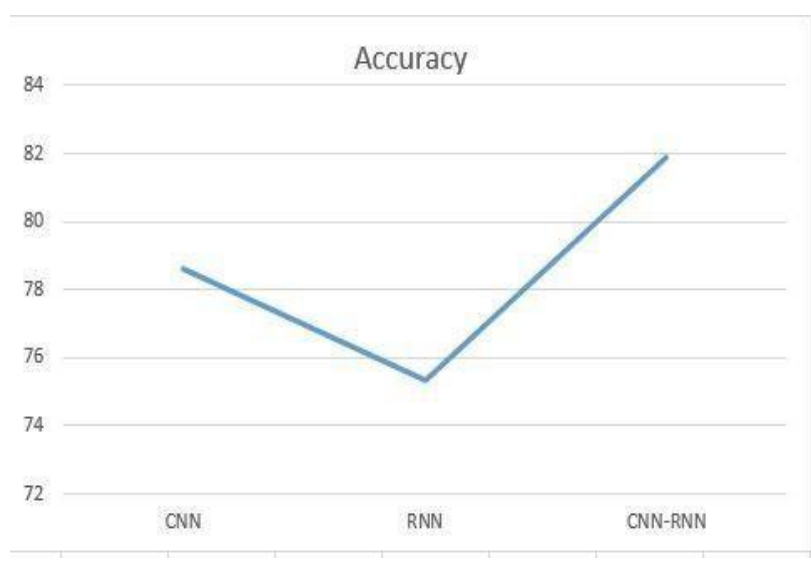

Fig 2: Accuracy comparison between CNN, RNN and CNN-RNN 


\section{CONCLUSION}

Sentiment Analysis is contextual mining of text which recognizes subjective information from the source material, and also assists the business that comprises the social sentiment of their service, brand or product observing chats. The goal is to calculate the sentiment accuracy of sentences that were extracted from the text of tweets. The sentiment analysis of the tweets helps to find whether the sentiment of the tweet on particular products, events, etc., is positive or negative. The challenging aspect in sentiment analysis is an opinion word which is considered as a positive in one situation may be considered as negative in another situation. The result shows comparison of sentiment classification using deep learning models. Accuracy is improved by combining the two deep learning models namely $\mathrm{CNN}$ and RNN. The hybrid model outperforms the individual $\mathrm{CNN}$ and RNN classification models with $81.90 \%$ accuracy. The model only relies on a pre- trained word vector representation model. As future work, will consider using different CNN architectures and try different ensemble models in order to improve the results. Also, explore using the distant training approach in order to train the word embeddings to reflect the sentiment polarity of the words. Additionally, verify the model over larger datasets other than Twitter dataset and compare the results with the recent state-of-the art deep learning model in tweets.

\section{REFERENCES}

[1] Yaser Maher Wazery, Hager Saleh Mohammed, EssamHalimHoussein, "Twitter Sentiment Analysis using Deep Neural Network", 14th Interna - tional Computer Engineering Conference (ICENCO), Cairo, Egypt, 2018, pp. 177-182.

[2] A. Ilmania, Abdurrahman, S. Cahyawijayaand A. Purwarianti, "Aspect Detection and Sentiment Classification Using Deep Neural Network for Indonesian Aspect-Based Sentiment Analysis,” 2018 International Conference on Asian Language Processing (IALP), Bandung, Indonesia, 2018, pp.62-67

[3] Merin Thomas, Latha C.A, "Sentimental analysis using recurrent neural network", International Journal of Engineering and Technology, Vol.7, No 2.27,2018.

[4] Ganda, Raouf, Mahmood, Ausif, "Sentiment Analysis With Re- Current Neural Network And Unsupervised Neural Language Model", 42nd International Conference on Acoustics, Speech and Signal Processing (ICASSP) 2017.

[5] P.Zhang,H.Zhu,T.XiongandY.Yang,"CoattentionNetworkandLow- rank Bilinear Pooling for Aspect Based Sentiment Analysis," ICASSP 2019 - 2019 IEEE International Conference on Acoustics, Speech and Signal Processing (ICASSP), Brighton, United Kingdom, 2019, pp. 6725- 6729.

[6] Y. Zhang, M. J. Er, R. Venkatesan, N. Wang and M. Pratama, "Sentiment classification using Comprehensive Attention Recurrent models," 2016 International Joint Conference on Neural Networks (IJCNN), Vancouver, BC, 2016, pp.1562-1569.

[7] F. Yang, C. Du, L. Huang, "Ensemble Sentiment Analysis Method based on R-CnnAnd C-Rnn With Fusion Gate", International Journal Of Computers Communications Control Issn1841-9836, E-Issn 18419844, 14(2), 272-285, April2019.
[8] C. Du, L. Huang, "Text Classification Research with Attention-based Recurrent Neural Networks", International Journal Of Com- Puters Communications Control Issn 1841-9836, 13(1), 50- 61, February2018.

[9] AsadAbdia, SitiMariyamShamsuddina, ShafaatunnurHasana, JalilPiranb, "Deep learning-based sentiment classification of evaluative text based on Multifeature fusion", Information Processing and Management, Vol. 56, Pg. 1245-1259,2019.

[10] MahaHeikal, Marwan Torki, Nagwa El-Makky, "Sentiment Analysis of Arabic Tweets using Deep Learning", The 4th International Conference on Arabic Computational Linguistics (ACLing 2018), November 17-19 2018

[11] G. Xu, Y. Meng, X. Qiu, Z. Yu and X. Wu, "Sentiment Analysis of Comment Texts Based on BiLSTM," in IEEE Access, vol. 7, pp. 51522- 51532,2019.

[12] Li, Liu, Zhang, "An Improved Approach for Text Sentiment Clas- sification Based on a Deep Neural Network via a Sentiment Attention Mechanism", Vol.11,2019.

[13] Akhtar, Md. Shad, AyushKumar, AsifEkbal and PushpakBhat- tacharyya. "A Hybrid Deep Learning Architecture for Sentiment Anal- ysis." COLING(2016).

[14] A. Cahyadi and M. L. Khodra, "Aspect-Based Sentiment Analysis Using Convolutional Neural Network and Bidirectional Long Short-Term Memory," 2018 5th International Conference on Advanced Informatics: Concept Theory and Applications (ICAICTA), Krabi, 2018 pp. 124-129, doi:10.1109/ICAICTA.2018.8541300.

[15] Zhang, Ziqi and Robinson, David and Tepper, Jonathan, "Detecting Hate Speech on Twitter Using a ConvolutionGRU Based Deep Neural Network", Springer International Publishing, 2018, pp. 745-760, isbn: 9783-319-93417-4

[16] D. Ekawati and M. L. Khodra, "Aspect-based sentiment analysis for indonesian restaurant reviews," in ICAICTA,2017.

[17] X. Ding, B. Liu and P. S. Yu, "A Holistic Lexicon-Based Approach to Opinion Mining," in Proceedings of the Conference on Web Search and Web Data Mining (WSDM),2008

[18] J. Pennington, R. Socher and C. D. Manning, "GloVe: Global Vectors for Word Representation," in The 2014 Conference on Empirical Methods in Natural Language Processing (EMNLP),2014.

[19] Z. Toh and J. Su, "NLANGP: Supervised Machine Learning System for Aspect Category Classification and Opinion TargetExtraction," in Proceedings of the 9th International Workshop on Semantic Evaluation (SemEval 2016), Denver,2015.

[20] A. Severyn and A. Moschitti, "UNITN: training deep convolutional neural Network for twitter snetiment classification," in Proceedings of the 9th International Workshop on Semantic Evaluation (SemEval 2015), Denver,2015

[21] P. Liu, S. Joty and H. Meng, "Fine-grained opinion mining with recurrent neural networks and word 
embeddings," in Proceedings of the 2015 Conference on Empirical Methods in Natural Language Processing, Lisbon,2015.A. N. Farhan and M. L. Khodra, "Sentiment-specific word embedding for Indonesian sentiment analysis," in ICAICTA,2017.

[22] Pennington, Jeffrey, Richard Socher, and Christopher D. Manning. "Glove: Global vectors for word representation." Proceedings of the Empiricial Methods in Natural Language Processing (EMNLP 2014) 12 (2014).

[23] Mikolov, Tomas, IlyaSutskever, Kai Chen, Greg S. Corrado, and Jeff Dean. "Distributed representations of words and phrases and their com- positionality." In Advances in Neural Information Processing Systems, pp. 3111-3119.2013.

[24] Svetlana Kiritchenko, Xiaodan Zhu, Colin Cherry, and Saif Mohammad, "Nrc-canada-2014: Detecting aspects and sentiment in customer reviews," in Proceedings of the8th International Workshop on Semantic Evaluation, 2014, pp.437-442.

[25] Duy-Tin Vo and YueZhang, "Target-dependent twitter sentiment clas- sification with rich automatic features.," in IJCAI, 2015, pp.1347-1353.

[26] Kai Sheng Tai, Richard Socher, and Christopher D Manning, "Improved semantic representations from tree structured long short-term memory networks," arXiv preprint arXiv:1503.00075,2015.

[27] Meishan Zhang, YueZhang, and Duy-Tin Vo, "Gated neural networks for targeted sentiment analysis.," inAAAI, 2016, pp. 3087-3093.

[28] Peng Chen, Zhongqian Sun, Lidong Bing, and Wei Yang, "Recurrent attention network on memory for aspect sentiment analysis," in Proceed- ings of the 2017 Conference on Empirical Methods in Natural Language Processing, 2017, pp.452-46. 